\title{
Differences in International Guidelines Regarding EMDR for Posttraumatic Stress Disorder: Why They Diverge and Suggestions for Future Research
}

\author{
Sarah Dominguez (1) \\ Murdoch University, Murdoch, Western Australia, Australia \\ Christopher W. Lee \\ The University of Western Australia, Crawley, Western Australia, Australia
}

\begin{abstract}
In the last 24 months, three separate practice guidelines for posttraumatic stress disorder (PTSD) have emerged from well-respected organizations that differed in the degree to which they recommend eye movement desensitization and reprocessing (EMDR) as a treatment. An international guideline was published by the International Society for Traumatic Stress Studies (ISTSS), and national guidelines were published by the American Psychological Association (APA) and the National Institute for Health Care Excellence (NICE). ISTSS reported that EMDR was effective and as potent as the best available therapies we can currently provide. NICE was more circumspect, and APA suggested other treatments had a stronger evidence base. In this review we focus on how these differences emerged and highlight the role of the time when the analysis was conducted, differences in inclusion criteria, and errors in determining appropriate measures. The 2017 APA guidelines were found to have the least validity when all these factors were considered. However, the fact that evaluating EMDR research is susceptible to such variations in methodology highlights certain research priorities that are then discussed.
\end{abstract}

Keywords: eye movement desensitization and reprocessing (EMDR); American Psychological Association; National Institute for Health Care Excellence; International Society for Traumatic Stress Studies; posttraumatic stress disorder (PTSD); evidence-based guidelines

0 fficial clinical guidelines for treatment of mental health conditions are designed to ensure that practitioners are able to easily navigate the evidence base and make an informed clinical decision. While cross-cultural differences are always acknowledged, psychology is an international community, and studies that are included, and the conclusions of these guidelines, are not limited to the continent where they originate. Therefore, we would expect evaluations of research outcomes conducted in different parts of the world to concur. A brief glance at recent recommendations from the International Society of Traumatic Stress Studies (ISTSS), the American Psychological Association (APA), and the National Institute for Health Care Excellence (NICE) for the use of eye movement desensitization and reprocessing (EMDR) for posttraumatic stress disorder (PTSD) seems to meet these expectations (Courtois et al., 2017; International Society of Traumatic Stress Studies, 2019; National Institute for Health Care Excellence, 2018). The three guidelines appear to correspond in that they propose that the research to date supports EMDR as an intervention for adults with PTSD. However, while the ISTSS offered strong support for the use of EMDR, the APA reported that the strength of the evidence was currently conditional but offered that it could possibly be upgraded to strong. In addition, while the NICE guidelines for the most part align with the ISTSS, they also identify caveats that were not identified in other guidelines, with regard to 
individuals who have experienced combat trauma and to children and adolescents.

The recommendations of each guideline are based primarily on meta-analyses. Prior to analysis, research questions are generated based on a predetermined population, intervention, comparison, and outcome (PICO) (Moher et al., 2015). This guides the inclusion and exclusion criteria that determine the available evidence that will be analyzed and how those studies will be examined. Most simply, meta-analyses are the pooled effectiveness of relevant studies with regard to the research questions (Borenstein, Hedges, Higgins, $\&$ Rothstein, 2011). In addition to the parameters in the PICO, studies are typically also evaluated for any methodical concerns that may increase the risk of bias, and studies that are seen to fall short are excluded. Risk of bias looks at the likelihood that a study may not be reporting on an actual treatment effect and may be influenced by another factor (Higgins et al., 2011). Such factors include poor randomization, failure to blind assessors, inappropriate assessment tools, selective reporting, and incomplete outcome data (Guyatt et al., 2011).

When the analysis is conducted, the strength of the evidence is evaluated as to the level of confidence that the estimated effect size of the intervention is correct. Analyses that included studies with poor design or with medium to high risk of bias are likely to have a lower strength of evidence rating (Higgins et al., 2011). A lower strength of evidence rating can also be given if the effect size from the analysis lacks precision (i.e., has a large confidence interval) or consistency (includes studies with conflicting results and a high unexplained heterogeneity), or are indirect (not directly related to the population in questions) (Puhan et al., 2014). Heterogeneity refers to differences in the intervention's outcomes across the included studies (Higgins \& Green, 2008).

Therefore, some of the differences between the guidelines might be explained by differences in the PICO and related constraints for inclusion of studies, or other parameters calculated such as the risk of bias. A brief summary of the inclusion and/or exclusion criteria pertaining to the PICO for each guideline is presented in the next section.

\section{Exclusion, Inclusion, and Population Studied in Each Guideline}

All guidelines included only randomized controlled trials (RCTs) that compared the trauma intervention with active controls, such as another trauma treatment, or inactive controls, such as wait-list or a placebo. Each review identified the psychological and pharmacological interventions that that were included in their analysis. In all three reviews, each intervention could to be delivered in conjunction with another intervention or as a stand-alone. Further, inclusions and exclusion criteria, as detailed in the relevant guide lines or accompanying documents, are outlined below.

\section{American Psychological Association}

The APA based their guidelines on a review conducted by the University of North Carolina Evidence Practice Center (UNCEPC) (Jonas et al., 2013). Their article included analysis only of adult participants who had been diagnosed with PTSD for more than 3 months. Further, the UNCEPC article was published in 2012 and therefore only articles available prior to this were included in their analysis. In addition, each study had to meet the following inclusion criteria:

- All participants must have a diagnosis of PTSD.

- Outcomes included PTSD symptom reduction, remission, prevention or reduction of comorbid symptoms, loss of PTSD diagnosis, quality of life, disability or functional impairment, return to work, and overall aversive effects of the intervention.

- Time period from 1980 to time of publication, to be updated after draft review.

- Any primary care, community, or military settings.

- Study duration greater than 4 weeks.

- Original research using randomized controlled trails design. No limits on sample size.

Exclusion criteria

- Children, risk of PTSD or subclinical populations.

- Interventions not identified prior to the analysis, including complementary or alternative approaches.

- All languages other than English.

- All non-RCTs, or studies deemed to have a high risk of bias.

APA Recommendations. The APA panel strongly recommended cognitive behavioral therapy (CBT), cognitive processing therapy (CPT), cognitive therapy $(\mathrm{CT})$, and prolonged exposure therapy (PE). Additionally, the panel offered conditional recommendation for use of brief eclectic psychotherapy (BEP), EMDR, and narrative exposure therapy (NET). However, they also stated that when the guidelines were updated beyond the current 2012 literature review, it was possible that the recommendations for EMDR and 
BEP would change to a strong rating. A summary of the recommendations is presented in Table 1.

\section{National Institute for Health Care Excellence}

The inclusion data for NICE was identical for adults and children. Studies with the mean age of participants over 18 were considered an adult study, while those with a mean age under 18 were considered to be studies on child and adolescents. The studies considered for analyses were based on the following inclusion criteria:

- Clinically important posttraumatic stress symptoms for more than 1 month after a traumatic event, defined by a diagnosis of PTSD according to Diagnostic and Statistical Manual of Mental Disorders (DSM), International Classification of Diseases (ICD), or similar criteria or clinically significant PTSD symptoms as indicated by baseline scores above threshold on a validated scale.

- At least $80 \%$ of the participants meet the diagnostic eligibility criteria.

- Studies are differentiated as early intervention ( $<3$ months) or delayed intervention (>3 months).
- Outcomes include efficacy; PTSD symptomology; PTSD diagnosis, remission, response, or relapse; and other important but not critical outcome measures.

- All RCTs are included. Unpublished data is included if risk of bias was assessed as adequate.

- Sample size at assessment point must be $n \geq 10$.

- Primary, secondary, tertiary, social care, or community settings.

Exclusion criteria

- Trials of people with adjustment disorders, traumatic grief, psychosis as a coexisting condition, learning disabilities, women with PTSD during pregnancy or in the first year following childbirth, or adults in contact with the criminal justice system (not solely as a result of being a witness or victim).

- Inoculation interventions for people who may be at risk of experiencing but have not experienced a traumatic event.

- Interventions that are not targeted at PTSD symptoms.

- Treatment provided to troops on operational deployment or exercise.

- Non-English articles, unless data can be obtained from an existing review.

- Studies with $<50 \%$ completion data.

TABLE 1. Summary of Guideline Recommendations

\begin{tabular}{|c|c|c|c|}
\hline & APA & NICE & ISTSS \\
\hline Adults PTSD & $\begin{array}{l}\text { Strong } \\
\text { recommendations: } \\
\text { CBT, CPT, CT, and PE. } \\
\text { Conditional for BEP, } \\
\text { EMDR, and NET. }\end{array}$ & $\begin{array}{l}\text { Recommendations: TF-CBT } \\
\text { (CPT, CT for PTSD, NET, and PE) } \\
\text { for presentations after } 1 \text { month; } \\
\text { EMDR for non-combat related } \\
\text { trauma after } 3 \text { months }\end{array}$ & $\begin{array}{l}\text { Strong recommendations-CPT, CT, } \\
\text { EMDR, Individual TF-CBT, and PE. } \\
\text { Standard recommendations-CBT, } \\
\text { Group TF-CBT, Guided Internet-based } \\
\text { TF-CBT, NET, and PCT. }\end{array}$ \\
\hline $\begin{array}{l}\text { Adults early } \\
\text { intervention }\end{array}$ & Not assessed & $\begin{array}{l}\text { Recommendations: TF-CBT } \\
\text { (CPT, CT for PTSD, NET, and PE) } \\
\text { for presentations before } 1 \text { month; } \\
\text { Conditional recommendation: } \\
\text { EMDR for non-combat trauma } \\
\text { (1-3 months post trauma) }\end{array}$ & $\begin{array}{l}\text { Standard recommendations-TF-CBT, } \\
\mathrm{CT} \text {, and EMDR }\end{array}$ \\
\hline $\begin{array}{l}\text { Children early } \\
\text { intervention }\end{array}$ & Not assessed & Recommendation: TF-CBT & Standard recommendation: TF-CBT \\
\hline Children PTSD & Not assessed & $\begin{array}{l}\text { Recommendation: TF-CBT } \\
\text { Conditional recommendation: } \\
\text { EMDR }\end{array}$ & $\begin{array}{l}\text { Strong recommendations for TF-CBT } \\
\text { and EMDR }\end{array}$ \\
\hline
\end{tabular}

Note APA = American Psychological Association; $\mathrm{BEP}=$ brief eclectic psychotherapy; $\mathrm{CBT}=$ cognitive behavioral therapy; $\mathrm{CPT}=$ cognitive processing therapy; $\mathrm{CT}=$ cognitive therapy; ISTSS $=$ International Society for Traumatic Stress Studies; NET = narrative exposure therapy; NICE = National Institute for Health Care Excellence; PCT = present-centered therapy; $\mathrm{PE}=$ prolonged exposure; TF-CBT; trauma-focused cognitive behavioral therapy. 
NICE Recommendations. For children who presented up to 3 months following a trauma, NICE recommended considering trauma-focused CBT (TFCBT) as an early intervention but stated that this was based on consensus clinical opinion, as there was not sufficient evidence to support this intervention against a control. For children with PTSD more than 3 months post trauma, NICE recommended TF-CBT, and considering EMDR only if children did not respond to or engage with TF-CBT. For early intervention for adults, NICE recommended offering TF-CBT (CPT, CT for PTSD, NET, and PE). For presentations after 1 month but before 3 months, NICE recommended considering EMDR for non-combat trauma if client has a preference for this, and otherwise to provide TF-CBT. For adults with PTSD for more than 3 months, NICE recommended TF-CBT or EMDR, unless it was combat related trauma, in which case offering TF-CBT was recommended. Finally, the NICE panel stated when TF-CBT was compared with EMDR directly for adult trauma, there was a non-significant trend toward EMDR being more efficacious and cost-effective (p. 37).

\section{International Society for Traumatic Stress Studies}

ISTSS did not identify any specific exclusion criteria. For the analysis ISTSS included the following:

- Any RCT (including cluster and crossover trials) evaluating the efficacy of interventions aimed at preventing, treating, or reducing symptoms of PTSD.

- The RCT is not solely a dismantling study.

- Individual, group, and couple interventions.

- No minimum sample size.

- Unpublished studies eligible.

Early interventions additional inclusions:

- Study participants have been exposed to a traumatic event as specified by PTSD diagnostic criteria for DSM-III, DSM-III-R, DSM-IV, DSM-5, ICD-9, ICD-10, or ICD-11.

- Intervention is not provided pre-trauma.

- Intervention begins no later than 3 months after the traumatic event.

- Study outcomes include a standardized measure of PTSD symptoms (either clinician-administered or self-report).

Treatment studies additional inclusions:

- At least $70 \%$ of participants required to be diagnosed with PTSD according to DSM or ICD criteria by means of a structured interview or diagnosis by a clinician. For children only, this can include partial PTSD (as defined as at least one symptom per cluster and presence of impairment), or score above a standard cutoff of a validated self-report measure.

- No restrictions on the basis of comorbidity, but PTSD required to be the primary diagnosis.

- Duration of PTSD symptoms required to be 3 months or more.

ISTSS Recommendations. For children, the ISTSS offered a standard recommendation for TF-CBT for early interventions. For children with PTSD, ISTSS gave a strong recommendation for TF-CBT (Child and Parent), TF-CBT Child, and EMDR. For early interventions with adults, TF-CBT, CT, and EMDR received standard recommendations. With regard to adults with PTSD, the ISTSS offered a strong recommendation for CPT, CT, EMDR, TF-CBT, and PE and standard recommendation for CBT without a trauma focus, group TF-CBT, guided internet-based TF-CBT, NET, and present-centered therapy. For a summary of all three reviews see Table 1.

\section{Comparison of the Guidelines}

As mentioned, while the APA guidelines provided recommendations only for adults with PTSD, NICE and ISTSS expanded their analysis to differentiate between established PTSD (3 or more months post trauma) and early treatment interventions (1-3 months post trauma) and prevention interventions $(<1$ month post trauma) for both adults and children. In addition to the differences in recommendations mentioned previously for adults with PTSD, disparities are evident in the conclusions about these more specific domains. For example, for early treatment interventions with adults, NICE offered support to several TF-CBT interventions and conditional support for the use of EMDR, while the ISTSS guidelines suggest that there is only standard evidence to support all interventions for this time period. In addition, within the recommendations for children with PTSD, ISTSS has strong recommendations for TF-CBT and EMDR, while NICE recommended TF-CBT and suggested considering EMDR only if the child does not respond or engage with TF-CBT.

\section{Evaluation of the Guidelines and Difference Between Them}

Tables 2-4 show all included studies in each guideline. For studies that have not been included in the organization's analysis, the reason for this is recorded if it was 
given or if easily deduced from the inclusion and exclusion criteria. Based on this analysis, there are several main factors that differ across the guidelines that contribute to the discord within the recommendations. A summary of these key points of difference are listed below.

\section{Time Frames for Literature Search}

It is obvious that some studies were excluded, as they were not available to the organizations at the time of the analysis. or leading up to the publication. Although APA, NICE, and ISTSS share publication date of their findings within 24 months of each other, the literature they survey varies considerably. NICE and ISTSS include articles published as recently as 2018, whereas the 2017 APA guidelines are restricted to articles published in 2012 or before. As shown in Table 2, this can account for why 11 studies could not be included in the APA analyses. This is important with regard to the findings of the APA, particularly in regard to the article by Van den Berg et al. (2015),

TABLE 2. Included and Excluded Studies of Adult PTSD by Review Organization, With Reason for Exclusion

\begin{tabular}{|c|c|c|c|}
\hline Adults Study & APA & NICE & ISTSS \\
\hline Acarturk $2015^{\mathrm{a}}$ & Published after analysis & Included & $\begin{array}{l}\text { Fell short of diagnostic } \\
\text { criteria }\end{array}$ \\
\hline Acarturk et al. (2016) & Published after analysis & Included & Included \\
\hline Ahmadi (2015) & Published after analysis & $\begin{array}{l}\text { Population outside } \\
\text { scope: Trials of soldiers } \\
\text { on active service }\end{array}$ & Included \\
\hline Aldahadha (2012) & Published after analysis & Included & $\begin{array}{l}\text { Fell short of diagnostic } \\
\text { criteria }\end{array}$ \\
\hline Capezzani et al. (2013) & Published after analysis & Included & Included \\
\hline Carletto (2016) & Published after analysis & Included & Included \\
\hline Carlson (1998) & Included & $\begin{array}{l}\text { Partially excluded due } \\
\text { to sample size } \\
(N<10 / \text { arm })\end{array}$ & Included \\
\hline Devilly $(1998)^{a}$ & $\begin{array}{l}\text { Fell short of diagnostic } \\
\text { criteria }\end{array}$ & $\begin{array}{l}\text { Non-randomized group } \\
\text { assignment }\end{array}$ & Included \\
\hline Devilly (1999) & High risk of bias & $\begin{array}{l}\text { Non-randomized group } \\
\text { assignment }\end{array}$ & Included \\
\hline $\begin{array}{l}\text { Edmond (1999) / } \\
\text { Edmond and Rubin } \\
(2004)^{\mathrm{a}}\end{array}$ & $\begin{array}{l}\text { Fell short of diagnostic } \\
\text { criteria }\end{array}$ & Included & $\begin{array}{l}\text { Fell short of diagnostic } \\
\text { criteria }\end{array}$ \\
\hline Himmerich et al. (2016) & Published after analysis & Included & Comparison not clear \\
\hline Hogberg (2007) & Included & $\begin{array}{l}\text { Sample size } \\
(N<10 / \text { arm })\end{array}$ & Included \\
\hline $\begin{array}{l}\text { Ironson, Freund, Strauss, } \\
\text { and Williams (2002) }\end{array}$ & High risk of bias & $\begin{array}{l}\text { Sample size } \\
(N<10 / \text { arm })\end{array}$ & Included \\
\hline Jensen $(1994)^{a}$ & Intervention too brief & Included & Intervention too brief \\
\hline Karatzias et al. (2011) & High risk of bias & Included & Included \\
\hline Laugharne et al. (2016); & Published after analysis & Included & Included \\
\hline Lee (2002) & High risk of bias & $\begin{array}{l}\text { Non-randomized group } \\
\text { assignment }\end{array}$ & Included \\
\hline Marcus (1997) & High risk of bias & Problems extracting data & Included \\
\hline Nijdam (2012) & $\begin{array}{l}\text { Reported in included studies } \\
\text { but not included in analysis }\end{array}$ & Included & Included \\
\hline
\end{tabular}

(Continued) 
TABLE 2. Included and Excluded Studies of Adult PTSD by Review Organization, With Reason for Exclusion (Continued)

\begin{tabular}{|c|c|c|c|}
\hline Adults Study & APA & NICE & ISTSS \\
\hline Power et al. $(2002)^{\mathrm{a}}$ & High risk of bias & Included & Included \\
\hline Rothbaum (1997) & Included & $\begin{array}{l}\text { Sample size } \\
(N<10 / \text { arm })\end{array}$ & Included \\
\hline Rothbaum (2005) & Included & Included & Included \\
\hline Scheck et al. $(1998)^{\star}$ & $\begin{array}{l}\text { Fell short of diagnostic } \\
\text { criteria }\end{array}$ & Included & Included \\
\hline Taylor et al. (2003) & Included & Included & Included \\
\hline $\begin{array}{l}\text { Ter Heide, Mooren, Van } \\
\text { de Schoot, De Jongh, and } \\
\text { Kleber }(2016)\end{array}$ & Published after analysis & Included & Not mentioned \\
\hline Van den Berg et al. (2015) & Published after analysis & $\begin{array}{l}\text { PTSD not primary } \\
\text { diagnosis }\end{array}$ & $\begin{array}{l}\text { PTSD not primary } \\
\text { diagnosis }\end{array}$ \\
\hline Van der Kolk et al. (2007) & Included but not analyzed & Included & $\begin{array}{l}\text { Placebo control not } \\
\text { accepted }\end{array}$ \\
\hline Vaughan $(1994)^{\star}$ & $\begin{array}{l}\text { Fell short of diagnostic } \\
\text { criteria }\end{array}$ & $\begin{array}{l}\text { Crossover study and first } \\
\text { phase data not available }\end{array}$ & Included \\
\hline Yurtsever et al. $(2018)^{\star}$ & Published after analysis & Included & $\begin{array}{l}\text { Fell short of diagnostic } \\
\text { criteria }\end{array}$ \\
\hline
\end{tabular}

Note. APA $=$ American Psychological Association; ISTSS = International Society for Traumatic Stress Studies; NICE = National Institute for Health Care Excellence; PTSD = posttraumatic stress disorder.

${ }^{a}$ Included participants with clinically important trauma symptoms. In other studies all participants had to meet full diagnostic criteria for PTSD.

TABLE 3. Included and Excluded Studies of Early Interventions for Trauma by Review Organization, With Reason for Exclusion

\begin{tabular}{|c|c|c|}
\hline Early Intervention Study & NICE & ISTSS \\
\hline Gil-Jardiné et al. (2018) & $\begin{array}{l}\text { Included in first month "prevention" } \\
\text { category }\end{array}$ & Included \\
\hline Jarero, Artigas, and Luber (2011) & Sample size $(N<10 /$ arm $)$ & Included \\
\hline $\begin{array}{l}\text { Jarero, Amaya, Givaudan, and } \\
\text { Miranda (2013) }\end{array}$ & Included & Not mentioned \\
\hline $\begin{array}{l}\text { Jarero, Uribe, Artigas, and } \\
\text { Givaudan (2015) }\end{array}$ & Not mentioned & Included \\
\hline Shapiro and Laub (2015) & Sample size $(N<10 /$ arm $)$ & Included \\
\hline $\begin{array}{l}\text { Shapiro, Laub, and Rosenblat } \\
\text { (2018) }\end{array}$ & Published after analysis & Included \\
\hline Tarquinio et al. (2016) & Not mentioned & Included \\
\hline
\end{tabular}

Note. ISTSS = International Society for Traumatic Stress Studies; NICE = National Institute for Health Care Excellence.

which is the largest randomized controlled trial ever published involving EMDR. This article was published 3 years prior to the APA publication yet was not included in the analysis. The APA authors identify that they were aware of the article and that it met their criteria. They did concede in the appendix, "The panel also concluded that, based on studies published between 2012 and June 2016, the recommendations 
TABLE 4. Included and Excluded Studies of Child and Adolescent PTSD by Review Organization, With Reason for Exclusion

\begin{tabular}{lll}
\hline Children Study & NICE & ISTSS \\
\hline $\begin{array}{l}\text { Ahmad (2007) } \\
\text { Chemtob, Nakashima, and Carlson (2002) }\end{array}$ & Included & Included \\
De Roos $(2017)^{\mathrm{a}}$ & Included & Included \\
Diehle (2015) & Not mentioned for EMDR; included for & Included \\
& other interventions & Included \\
Jaberghaderi, Greenwald, Rubin, Zand, and & Sample size $(N<10 /$ arm $)$ & Included \\
Dolatabadi $(2004)^{\mathrm{a}}$ & & PTSD not primary \\
Kemp $(2010)^{\mathrm{a}}$ & Data could not be extracted & diagnosis \\
\hline
\end{tabular}

Note. ISTSS = International Society for Traumatic Stress Studies; NICE = National Institute for Health Care Excellence; PTSD = posttraumatic stress disorder.

${ }^{a}$ Included participants with clinically important trauma symptoms. In other studies all participants had to meet full diagnostic criteria for PTSD.

for EMDR... may change from conditional ('the panel suggests') to strong ('the panel recommends.)" (Courtois et al., 2017, p. ii); however, this is not part of the main body of the report.

Further evidence to support the argument that this time lag between analysis and publication accounts for the differences in the reviews comes from an updated analysis by UNCEPC (Forman-Hoffman et al., 2018). UNCEPC reviewed their 2012 analysis, which formed the basis for the APA's 2017 guidelines, and then updated this with more recent published articles and articles that had previously been omitted. In the updated review, three more recent studies were added (Acarturk et al., 2016; Ter Heide et al., 2016; Van den Berg et al., 2015) as well as an older study that had not appeared in the original analysis (Van der Kolk et al., 2007). The inclusion of these studies lowered the heterogeneity (i.e., the estimated treatment effect size for EMDR was judged to be more consistent), which caused a revision to the strength of evidence from low to moderate. This updated review, if adopted by the APA, would bring its guidelines on EMDR closer to those of ISTSS and NICE.

\section{Included Population}

While all three guidelines purport to investigate adult PTSD, they differ on the criteria as to what this means. APA specified that all participants must meet diagnostic criteria for PTSD. By far this is the most restrictive criteria and not typical of most international guidelines. In contrast, ISTSS specified that a study would be included if $70 \%$ of participants were required to be diagnosed with PTSD, whereas NICE specifies that at least $80 \%$ have a diagnosis of PTSD or clinically significant PTSD symptoms as indicated by diagnosis or baseline scores above threshold on a validated scale. The differences in the inclusion criteria with regard to PTSD diagnosis account for some of the discrepancies in the included and excluded studies. For example, it is why Scheck et al. (1998) is excluded from APA but included in ISTSS and NICE. Reliance on participants who have been formally diagnosed with PTSD ensures the study is directly related to the population of interest. However, more liberal inclusion of trauma populations such as that used by ISTSS ensures that as many possible studies that are relevant to the area of interest are included.

Reliance on a binary classification system-that is, the presence or absence of a PTSD diagnosismeans more confidence in the interpretation of a study when clinicians to know if a particular treatment is applicable to a client who has that diagnosis. However, clinicians do not only treat people with a clear diagnosis. Clinicians are likely to treat someone with PTSD even if they do not meet $100 \%$ of the diagnostic criteria. If, following a trauma event, a client presents with a combination of the intrusions, avoidance, negative mood/cognitions, and alterations in arousal and reactivity, the clinician will treat this symptom cluster. This is why many clinicians and academics are now arguing for the adoption of a dimensional rather than categorical diagnostic system (Brown \& Barlow, 2005; Widiger \& Samuel, 2005).

It is interesting that in their most recent review, UNCEPC no longer restricts their analysis to only 
studies where all participants meet the PTSD criteria. In their 2018 update, they amended their inclusion criteria to include any study, provided "'most" participants met PTSD criteria, which brings them into line with ISTSS and NICE.

There is similar discord with regard to comorbid conditions. ISTSS stated that PTSD diagnosis had to be the primary diagnosis, and NICE excluded any article where psychosis was a comorbid condition. This accounts for each of these guidelines' exclusion of the Van den Berg et al. (2015) article. Again, it appears that this restriction may support the applicability of the reviews to the population in question. However, this restriction may limit the generalizability of the guidelines to patients typically seen in practice, given that $80 \%$ of individuals with PTSD also meet criteria for another mental health condition (Galatzer-Levy, Nickerson, Litz, \& Marmar, 2013). Identifying which is the primary disorder can be complex and is typically subjective, based on both the patient and clinician particulars. Further, several studies were included for analysis when PTSD was clearly a secondary diagnosis, such as looking at oncology patients (Capezzani et al., 2013). Providing information to clinicians about studies where comorbid conditions are included may better inform everyday practice.

\section{Number of Participants}

As can be seen in Tables 2-4, the NICE guidelines exclude several articles (Högberg et al., 2008; Ironson et al., 2002; Jaberghaderi et al., 2004; Jarero et al., 2011; Rothbaum, 1997; Shapiro \& Laub, 2015), as the final number of participants in at least one of the treatment conditions fell below 10 . With regard to studies examining EMDR, this results in the exclusion of a total of 104 participants. The exclusion of trials is common practice when a rapid rather than a comprehensive review is needed (Turner, Bird, \& Higgins, 2013). In more comprehensive approaches to meta-analysis, all available valid data is used to gain an overall understanding of the current state of evidence, and the number of participants in each group is taken into consideration in the analysis.

In addition to excluding the studies in the above paragraph, some partial exclusions also occurred in the NICE analysis with regard to follow-up data. The Carlson study (1998) examined treatments for combatrelated PTSD and used the Clinician-Administered PTSD Scale (CAPS) to assess PTSD diagnosis at follow-up. This assessment was conducted 3 months post treatment, and only 9 participants attended the assessment from each treatment group. This data was ignored because although originally there were more than 10 participants per cell, by follow-up this was down to nine. Of the 9 participants who received biofeedback, 7 continued to meet criteria for PTSD 3 months after treatment had completed, whereas only of the participants who received EMDR continued to do so. When the number of studies are few and the sample sizes small, such restrictions can account for why treatments are supported in one review but not in others. It also highlights for EMDR the need to conduct further research in this area and with larger sample sizes.

\section{Unclear/ Erroneous Study Exclusions}

Several articles were excluded from the NICE article with no explanation or insufficient justification. This is likely to have had a systematic effect on why the recommendations for EMDR with children were stronger for the ISTSS guidelines compared to the NICE guidelines. An example of two studies included in the ISTSS report but not mentioned in the NICE article are Chemtob et al. (2002) and Tarquinio et al. (2016). The Chemtob article reported on the findings of treating 32 children with PTSD who were randomly assigned to EMDR or wait-list treatment conditions, and the Tarquinio article reported on 60 adults who received early psychological interventions following workplace trauma. The NICE review does not mention either study on either its exclusion or inclusion list. The Kemp (2010) article was also excluded from the NICE analysis, as the NICE researchers reported they were unable to extract the necessary data. While this might be true for the published journal article of the Kemp study, the data is readily available on an earlier version of the study published via a university repository. That is probably why the ISTSS researchers were able to extract the data. There were similar issues with some adult studies. Marcus and colleagues found significant improvement for EMDR over a wait list for 67 people and that the benefits were stable at 6 months follow-up (Marcus, Marquis, $\&$ Sakai, 2004). This study was excluded from NICE analysis. The researchers stated "efficacy or safety data cannot be extracted (p. 1225, appendix D) yet the ISTSS researchers were able to extract this data.

Another article that is oddly excluded or partially excluded from all three guidelines is a 2007 study by Van der Kolk et al. that examined the difference between EMDR, SSRI medication, and a placebo control. This study was included in the analysis in the APA article in the medication sections, but the data was inexplicably excluded from the analysis of EMDR 
with a control condition. As outlined in Dominguez and Lee (2017), the inclusion of this article would have made a significant difference to final recommendation for EMDR. Interestingly, this error was rectified in the revised analysis conducted by UNCEPC. In the NICE article, the study is analyzed; however, results do not appear to be considered in their recommendations, while the ISTSS does not mention the article at all.

\section{Incorrect Measures Chosen}

In the Carlson et al. (1998) study, all three reviews used a measure incongruent to the other data in the study. The Impact of Event Scale (IES) was chosen to assess change; however, the Mississippi Scale for Combat-Related PTSD (M-PTSD) was the more appropriate measure (see Dominguez \& Lee, 2017 for more detailed analysis). While the IES results were not significant, changes in the M-PTSD post treatment were, and the effect size at follow-up compared to the other treatment conditions was large $(d>1.01)$. At the time of the Carlson publication, the M-PTSD was recommended above all other self-report measures for assessing PTSD symptoms in veterans (Watson, 1990). Further, the improvement on the M-PTSD measure was similar in effect size to the improvement in the CAPS, providing further validity that the MPTSD was the more appropriate measure. Recalculating the effect size in the meta-analysis has been shown to change the recommendations for PTSD in the APA guidelines (Dominguez \& Lee, 2017) and so would also likely change the outcome in the NICE analysis with respect to combat PTSD.

In meta-analyses two researchers typically read a study and then determined what measure to assess outcome by and what figures to enter as the results. While this protects from errors, it does not guarantee that correct measures and figures are chosen. Another example of this is in the UNCEPC analysis with regard to Van der Kolk et al. (2007). As mentioned, this study was excluded in 2012 but included in 2018, although in the recent version the authors used the wrong values to compare EMDR to the placebo, citing the effect (standard mean difference) as .09 when it should be .65 .

\section{Putting It All Together: What Can We Take Away for APA, NICE, and ISTSS?}

In this section we focus on how the above differences in examining the outcome literature have impacted the guidelines and what the take-home message is for clinicians. Beginning with the 2017 APA guidelines, it is clear from the above that most of the difference in its recommendations from the other two guidelines can be accounted for by the restriction of the analysis to literature published before 2012. A second issue is the narrow interpretation of what constitutes a study on PTSD. Of the three guidelines, the APA has the most restrictive approach to this inclusion criterion. Recent revisions of the literature by UNCEPC have attempted to correct this by both broadening the definition of PTSD and by including more recent studies. Given these developments, the rating of EMDR as evidence based was strengthened in the revised analysis, rendering the APA 2017 guidelines outdated. There are other oddities in the APA guidelines that have been identified in prior articles, such as the inconsistencies in the way the strength of evidence was assessed across different intervention types and the way that study bias was assessed (see Dominguez \& Lee, 2017 for more details).

In contrast to the APA, the NICE guidelines' period of evidence review was closer to the guideline publication date, and their inclusion criteria meant they examined studies where PTSD was the major symptom cluster, which probably more typically represents what clinicians usually are asked to treat. So overall, the NICE guidelines are more applicable to clinical practice now compared to the APA. Although the findings generally line up with ISTSS, they differ with respect to three subgroups: children, early intervention, and combat PTSD. The above discussion of inclusion/exclusion criteria highlights why these differences occurred. NICE was found to favor studies with a large sample size, which is an approach suitable for quick reviews. The effect of this decision has particular implications for the evaluation of EMDR, as many of the studies were clinician-focused research rather than institution-focused research, which tends to utilize larger numbers. Secondly, some differences between the NICE and ISTSS guidelines appeared because the NICE researchers were not able to calculate effect sizes for some studies favorable to EMDR, whereas ISTSS researchers were.

With respect to early intervention, the ISTSS guidelines offer standard support for the use of EMDR for individuals up to 3 months after the traumatic event, and the NICE guidelines make a conditional recommendation for its use during the second and third month. For the NICE analysis, this is based on a single study (Jarero et al., 2013). The ISTSS guidelines examined six studies overall and looked separately at singlesession early intervention (Gil-Jardiné et al., 2018; Tarquinio et al., 2016) and early treatment interventions (Jarero et al., 2015; Shapiro \& Laub, 2015; 
Shapiro et al., 2018), with one study in both analyses (Jarero et al., 2011). The differences in study inclusions is partly explicable by considering the NICE exclusion criteria highlighted above, such as when the guidelines were published, and excluding studies with less than 10 participants in each arm. The impact of these extra studies in the ISTSS guidelines led to the finding of a large effect size for EMDR (standard mean difference $[\mathrm{SMD}]=2.50$ ); however, the variability in the type of interventions compared led to relatively large heterogeneity $\left(\mathrm{I}^{2}=88 \%\right)$, which decreases the confidence in the final analysis.

NICE guidelines also did not recommend EMDR for use with combat veterans. This was based on three studies that were assessed as providing evidence of EMDR's ability to change PTSD symptoms (Carlson, Chemtob, Rusnak, \& Hedlund, 1996; Himmerich et al., 2016; Jensen, 1994). In the analysis each study showed a non-significant effect for EMDR compared to the comparison condition. While this may reflect the current state of evidence for EMDR with combat veterans, the conclusion may not reflect the suitability of EMDR for this population group. The inclusion of the Jensen (1994) study, which compared EMDR as an add-on to existing veteran service treatment, is an issue in that the participants had only two sessions of EMDR therapy. The ISTSS initially included this study; however, they then removed it from their analysis, as they stated administering only two sessions is not delivering the intervention as intended, thus is not an accurate measure of the intervention efficacy. APA also excluded this study for a similar reason. The second study was Carlson et al. (1998), in which, as described above, an inappropriate measure was chosen to assess its efficacy, inaccurately suggesting that the participants did not get better. In the review above, we point out that rather than EMDR not helping, the effect size was large for more appropriate measures such as the CAPS or M-PTSD. Other evidence to suggest EMDR may yet prove effective for combat trauma comes from a German study where 40 veterans were randomized to either Imagery Rescripting or EMDR (Alliger-Horn, Zimmermann, \& Mitte, 2015). Both groups improved with the Reliable Change Index for EMDR at $77 \%$ and $67 \%$ for Imagery Rescripting.

The NICE meta-analysis's focus on combat-related PTSD in a subanalysis is an important initiative, given the typical lower effect sizes found for this population. In the analysis, TF-CBT was found to lead to a significant improvement compared to treatment as usual (p. 592, appendix D). This was based on three studies, but only two of the studies found a significant difference. TF-CBT performs less well when compared to an active treatment. The NICE guidelines include a fourth study where TF-CBT was compared to present-centered therapy (Schnurr et al., 2003), and the resultant difference was negligible $(\mathrm{SMD}=.07)$. In an even larger study of 370 veterans published in January 2018 (Foa et al., 2018) but not included in the NICE guidelines, the differences between prolonged exposure and present-centered therapy were also negligible $(d=0.1)$. Given these results, there is a clear need for further research on the effectiveness of TF-CBT in treating veterans with PTSD; although in many of these studies people experience some symptom improvement, the actual percentage of people still meeting diagnostic criteria for PTSD at follow-up is 60\% (Foa et al., 2018; Monson et al., 2006).

\section{Conclusions and Future Directions}

The above review highlighted key differences between the three guidelines (APA, NICE, and ISTSS) and the likely basis that resulted in these differences. While there were clear methodological differences in the APA analysis, differences within the other two guidelines have helped to identify important research gaps. These gaps relate to both research design issues and the type of trauma populations that need more research.

\section{Use of Wait-List Comparisons}

A problem with existing research on EMDR is that it has focused on comparisons with active treatments. In the ISTSS guidelines there were 51 studies listed where TF-CBT was compared to a wait-list control. However, there were only 24 trials where TF-CBT was compared to another non-CBT active treatment. Therefore, $68 \%$ of all TF-CBT trials involve a wait list.

Using the ISTSS guidelines, the ratio of waitlist comparisons to active treatments is very different for EMDR. The total number of EMDR studies where EMDR was compared to wait list was 11, whereas there were 17 trials where EMDR compared to another active treatment. Therefore, only $39 \%$ of all EMDR trials involve a wait list. The problem with this focus on active treatments is that it is likely to lower the perceived advantage of providing EMDR. Not surprisingly, meta-analysis of PTSD research has found that studies involving comparisons of active treatment to another treatment for PTSD will show a lower benefit than when an active treatment is compared to a wait-list control (Karatzias et al., 2019). 
Additional Research for Children, Early Interventions, and Combat Veterans

The above review also highlighted a difference between NICE and ISTSS guidelines with respect to the recommendations for children. While there is an argument that some studies should have been included in the NICE guidelines and that this would likely have resulted in a higher recommendation equivalent to ISTSS, the fact that two or three missing studies impact the analysis underscores the importance of further trials in this area.

An identical issue applies for the research for early interventions where the failure to include some studies lowered the evidence level for NICE compared to ISTSS. ISTSS found that EMDR was effective in helping to prevent PTSD symptoms in people recently exposed to a traumatic event. NICE did not include five studies that were in the "ISTSS" analysis (see Table 4), which explains the discrepancy. However, the studies in this area used very different doses and protocols, leading to high variability in the outcomes. Replications of these studies with larger samples should enable improved confidence in recommending this type of intervention. In addition, there is not one single RCT examining the use of EMDR early intervention with children or adolescents, and such research should be given high priority.

NICE conducted a subanalysis that highlighted issues with treating combat veterans. EMDR was not recommended for this group of patients. The number of studies assessing TF-CBT and EMDR with this population is very small. This highlights the need for further research in this area. For this group of patients it is clear that all treatment approaches need to improve. Even a therapy that was recommended in the NICE guidelines (TF-CBT) appears no better than an alternative active treatment, and most veterans still have PTSD at the end of treatment.

\section{Quality of Research Design}

Finally, a comment on the quality of research design. This review highlighted the need to further investigate the use of EMDR with a waitlist control designs and to also focus on special populations such as children, veterans, and early interventions. Researchers that do this need to pay attention to research quality to ensure that their studies are included in future reviews and not excluded because they are assessed as possibly biased. This can be done by paying attention to guidance offered in review articles on methodology (Maxfield \& Hyer, 2002) or by accessing resources such as CONSORT (Schulz, Altman, \& Moher, 2010).

\section{References}

Acarturk, C., Konuk, E., Cetinkaya, M., Senay, I., Sijbrandij, M., Cuijpers, P., \& Aker, T. (2015). EMDR for Syrian refugees with posttraumatic stress disorder symptoms: Results of a pilot randomized controlled trial. European Journal of Psychotraumatology, 6, 9. doi:10.3402/ ejpt. v6.27414

Acarturk, C., Konuk, E., Cetinkaya, M., Senay, I., Sijbrandij, M., Gulen, B., \& Cuijpers, P. (2016). The efficacy of eye movement desensitization and reprocessing for post-traumatic stress disorder and depression among Syrian refugees: Results of a randomized controlled trial. Psychological Medicine, 46, 1-11. doi:10.1017/S0033291716001070

Ahmad, A., Larsson, B., \& Sundelin-Wahlsten, V. (2007). EMDR treatment for children with PTSD: Results of a randomized controlled trial. Nordic Journal of Psychiatry, 61(5), 349-354. doi:10.1080/08039480701643464

Ahmadi, K., Hazrati, M., Ahmadizadeh, M., \& Noohi, s. (2015). REM desensitization as a new therapeutic method for post-traumatic stress disorder: A randomized controlled trial. Acta Med Indones, 47(2), 111-119.

Aldahadha, B., Al-Harthy, H., \& Sulaiman, S. (2012). The efficacy of eye movement desensitization reprocessing in resolving the trauma caused by the road accidents in the Sultanate of Oman. Journal of Instructional Psychology, 39(3-4), 146-158.

Alliger-Horn, C., Zimmermann, P., \& Mitte, K. (2015). Vergleichende Wirksamkeit von IRRT und EMDR bei kriegstraumatisierten deutschen Soldaten. Trauma und Gewalt, 9(3), 204-215. Retreived from http:// www.traumaundgewalt.de/article/tg_2015_03_02040215_0204_01

Borenstein, M., Hedges, L. V., Higgins, J. P., \& Rothstein, H. R. (2011). Introduction to meta-analysis. West Sussex, United Kingdom: John Wiley \& Sons.

Brown, T. A., \& Barlow, D. H. (2005). Dimensional versus categorical classification of mental disorders in the fifth edition of the Diagnostic and Statistical Manual of Mental Disorders and beyond: Comment on the special section. Journal of Abnormal Psychology, 114(4), 551. doi:10.1037/0021-843X.114.4.551

Capezzani, L., Ostacoli, L., Cavallo, M., Carletto, S., Fernandez, I., Solomon, R., \& Cantelmi, T. (2013). EMDR and CBT for cancer patients: Comparative study of effects on PTSD, anxiety, and depression. Journal of EMDR Practice and Research, 7(3), 134-143. doi:10.1891/1933-3196.7.3.134

Carletto, S., Borghi, M., Bertino, G., Oliva, F., Cavallo, M., Hofmann, A., . . . Ostacoli, L. (2016). Treating post-traumatic stress disorder in patients with multiple sclerosis: A randomized controlled trial comparing the efficacy of eye movement desensitization and reprocessing and relaxation therapy. Frontiers in Psychology, 7, 526. doi:10.3389/fpsyg.2016.00526

Carlson, J. G., Chemtob, C. M., Rusnak, K., \& Hedlund, N. L. (1996). Eye movement desensitization and 
reprocessing treatment for combat-related posttraumatic stress disorder. Psychotherapy, 33, 104-113. doi:10.1037/0033-3204.33.1.104

Carlson, J. G., Chemtob, C. M., Rusnak, K., Hedlund, N. L., \& Muraoka, M. Y. (1998). Eye movement desensitization and reprocessing (EDMR) treatment for combat-related posttraumatic stress disorder. Journal of Traumatic Stress, 11(1), 3-24. doi:10.1023/A:1024448814268

Chemtob, C. M., Nakashima, J., \& Carlson, J. G. (2002). Brief treatment for elementary school children with disaster-related posttraumatic stress disorder: A field study. Journal of Clinical Psychology, 58(1), 99-112. doi:10.1002/jclp. 1131

Courtois, C. A., Brown, L. S., Cook, J., Fairbank, J. A., Friedman, M., Gone, J. P., \& Roberts, J. (2017). Clinical practice guideline for the treatment of posttraumatic stress disorder (PTSD) in adults. Retrieved from https: / / www.apa.org/about/offices/directorates/guidelines / ptsd.pdf.

De Roos, C., Van der Oord, S., Zijlstra, B., Lucassen, S., Perrin, S., Emmelkamp, P., \& De Jongh, A. (2017). Comparison of eye movement desensitization and reprocessing therapy, cognitive behavioral writing therapy, and wait-list in pediatric posttraumatic stress disorder following single-incident trauma: A multicenter randomized clinical trial. Journal of Child Psychology and Psychiatry, 58(11), 1219-1228. doi:10.1111/jcpp.12768

Devilly, G. J., \& Spence, S. H. (1999). The relative efficacy and treatment distress of EMDR and a cognitivebehavior trauma treatment protocol in the amelioration of posttraumatic stress disorder. Journal of Anxiety Disorders, 13(1-2), 131-157. doi:http://dx.doi.org/ 10.1016/S0887-6185(98)00044-9

Devilly, G. J., Spence, S. H., \& Rapee, R. M. (1998). Statistical and reliable change with eye movement desensitization and reprocessing: Treating trauma within a veteran population. Behavioral Therapy, 29(3), 435-455. http: / / dx.doi.org/10.1016/s0005-7894(98)80042-7

Diehle, J., Opmeer, B. C., Boer, F., Mannarino, A. P., \& Lindauer, R. J. (2015). Trauma-focused cognitive behavioral therapy or eye movement desensitization and reprocessing: What works in children with posttraumatic stress symptoms? A randomized controlled trial. Eur Child Adolesc Psychiatry, 24(2), 227-236. doi:10.1007/s00787-0140572-5 http: / / dx.doi.org/10.1007/ s00787-014-0572-5

Dominguez, S. K., \& Lee, C. W. (2017). Errors in the 2017 APA clinical practice guideline for the treatment of PTSD: What the data actually says. Frontiers in Psychology, 8, 1425. doi:10.3389/fpsyg.2017.01425

Edmond, T., \& Rubin, A. (2004). Assessing the long-term effects of EMDR: Results from an 18-month follow-up study with adult female survivors of CSA. Journal of Child Sexual Abuse, 13(1), 86. http: / / dx.doi.org/10.1300/ j070v13n01_04

Edmond, T., Rubin, A., \& Wambach, K. G. (1999). The effectiveness of EMDR with adult female survivors of childhood sexual abuse. Social Work Research, 23(2), 116. doi:http: / dx.doi.org/10.1093/swr/23.2.103
Foa, E. B., McLean, C. P., Zang, Y., Rosenfield, D., Yadin, E., Yarvis, J. S., \& Dondanville, K. A. (2018). Effect of prolonged exposure therapy delivered over 2 weeks vs 8 weeks vs present-centered therapy on PTSD symptom severity in military personnel: A randomized clinical trial. JAMA, 319(4), 354-364. doi:10.1001/jama.2017.21242

Forman-Hoffman, V., Middleton, J. C., Feltner, C., Gaynes, B. N., Weber, R. P., Bann, C., . . . Green, J. (2018). Psychological and pharmacological treatments for adults with posttraumatic stress disorder: A systematic review update. Rockville, MD: Agency for Healthcare Research and Quality. Retrieved from https: / www.ncbi.nlm.nih.gov/books/NBK525132/

Galatzer-Levy, I. R., Nickerson, A., Litz, B. T., \& Marmar, C. R. (2013). Patterns of lifetime PTSD comorbidity: A latent class analysis. Depression and Anxiety, 30(5), 489-496. doi:10.1002/ da.22048

Gil-Jardiné, C., Evrard, G., Joboory, Al., S., Saint Jammes, T, J., Masson, F., ... Régis, C. A. (2018). Emergency room intervention to prevent post concussion-like symptoms and post-traumatic stress disorder. A pilot randomized controlled study of a brief eye movement desensitization and reprocessing intervention versus reassurance or usual care. Journal of Psychiatric Research, 103, 229-236. doi:10.1016/j.jpsychires.2018.05.024

Guyatt, G. H., Oxman, A. D., Vist, G., Kunz, R., Brozek, J., Alonso-Coello, P., \& Falck-Ytter, Y. (2011). GRADE guidelines: 4. Rating the quality of evidence-Study limitations (risk of bias. Journal of Clinical Epidemiology, 64(4), 407-415. doi:10.1016/j.jclinepi.2010.07.017

Högberg, G., Pagani, M., Sundin, Ö, Soares, J., ÅbergWistedt, A., Tärnell, B., \& Hällström, T. (2008). Treatment of post-traumatic stress disorder with eye movement desensitization and reprocessing: Outcome is stable in 35-month follow-up. Psychiatry Research, 159 (1-2), 101-108. doi:10.1016/j.psychres.2007.10.019

Higgins, J. P., Altman, D. G., Gøtzsche, P. C., Jüni, P., Moher, D., Oxman, A. D., \& Sterne, J. A. (2011). The Cochrane collaboration's tool for assessing risk of bias in randomised trials. BMJ, 343, d5928. doi:10.1136/bmj.d5928

Higgins, J. P., \& Green, S. (2008). Cochrane handbook for systematic reviews of interventions. West Sussex, England: The Cochrane Collaboration and John Wiley \& Sons Ltd.

Himmerich, H., Willmund, G. D., Zimmermann, P., Wolf, J.-E., Bühler, A., C Kirkby, K ., . . . Wesemann, U. (2016). Serum concentrations of TNF-a and its soluble receptors during psychotherapy in German soldiers suffering from combat-related PTSD. Psychiatria Danubina, 28(3), 293-298.

International Society of Traumatic Stress Studies. (2019). Posttraumatic stress disorder. Prevention and treatment guidelines. Retrieved from www.istss.org/treatingtrauma/new-istss-prevention-and-treatment-guidelines

Ironson, G., Freund, B., Strauss, J., \& Williams, J. (2002). Comparison of two treatments for traumatic stress: A community-based study of EMDR and prolonged 
exposure. Journal of Clinical Psychology, 58(1), 113-128. doi:10.1002/jclp.1132

Jaberghaderi, N., Greenwald, R., Rubin, A., Zand, S. O., \& Dolatabadi, S. (2004). A comparison of CBT and EMDR for sexually-abused Iranian girls. Clinical Psychology \& Psychotherapy, 11(5), 358-368. doi:10.1002/cpp.395

Jarero, I., Amaya, C., Givaudan, M., \& Miranda, A. (2013). EMDR individual protocol for paraprofessional use: A randomized controlled trial with first responders. Journal of EMDR Practice and Research, 7(2), 55-64. doi:10.1891/1933-3196.7.2.55

Jarero, I., Artigas, L., \& Luber, M. (2011). The EMDR protocol for recent critical incidents: Application in a disaster mental health continuum of care context. Journal of EMDR Practice and Research, 5(3), 82-94. doi: 10.1891/1933-3196.5.3.82

Jarero, I., Uribe, S., Artigas, L., \& Givaudan, M. (2015). EMDR protocol for recent critical incidents: A randomized controlled trial in a technological disaster context. Journal of EMDR Practice and Research, 9(4), 166. doi:10.1891/1933-3196.9.4.166

Jensen, J. A. (1994). An investigation of eye movement desensitiztion and reprocessing $(\mathrm{EMD} / \mathrm{R})$ as a treatment for posttraumatic stress disorder (PTSD) syptoms of Vietnam combat veterans. Behavior Therapy, 25, 311-326. doi:10.1016/S0005-7894(05)80290-4

Jonas, D. E., Cusack, K., Forneris, C. A., Wilkins, T. M., Sonis, J., Middleton, J. C., \& Brownley, K. A. (2013). Psychological and pharmacological treatments for adults with posttraumatic stress disorder (PTSD). Rockville, MD: AHRQ.

Karatzias, T., Murphy, P., Cloitre, M., Bisson, J., Roberts, N., Shevlin, M., . . . Hutton, P. (2019). Psychological interventions for ICD-11 complex PTSD symptoms: Systematic review and meta-analysis. Psychological Medicine, 49, 1761-1775. doi:10.1017/S0033291719000436

Karatzias, T., Power, K., McGoldrick, T., Begum, M., Young, J., \& Adams, S. (2011). A controlled comparison of the effectiveness and efficiency of two psychological therapies for posttraumatic stress disorder: Eye movement desensitization and reprocessing vs. emotional freedom techniques. Journal of Nervous and Mental Disease, 199(6), 372-378. doi:http: / / dx.doi.org/10.1097/nmd.0b013e31821cd262

Kemp, M., Drummond, P., \& McDermott, B. (2010). A wait-list controlled pilot study of eye movement desensitization and reprocessing (EMDR) for children with post-traumatic stress disorder (PTSD) symptoms from motor vehicle accidents. Clinical Child Psychology and Psychiatry, 15(1), 5-25. doi:http://dx.doi.org/10.1177/ 1359104509339086

Laugharne, J., Kullack, C., Lee, C.W., McGuire, T., Brockman, S., Drummond, P., \& Starkstein, S. (2016). Amygdala volumetric change following psychotherapy for posttraumatic stress disorder. Journal of Neuropsychiatry and Clinical Neurosciences, 28(4), 312-318. http: / / dx. doi.org/10.1176/appi.neuropsych.16010006
Lee, C. W., Gavriel, H., Drummond, P., Richards, J., \& Greenwald, R. (2002). Treatment of PTSD: Stress inoculation training with prolonged exposure compared to EMDR. Journal of Clinical Psychology, 58(9), 1071-1089. doi:10.1002/jclp.10039

Marcus, S. V., Marquis, P., \& Sakai, C. (1997). Controlled study of treatment of PTSD using EMDR in an HMO setting. Psychotherapy, 34(3), 307-315. doi:10.1037/h0087791

Marcus, S., Marquis, P., \& Sakai, C. (2004). Three- and 6-month follow-up of EMDR treatment of PTSD in an HMO setting. International Journal of Stress Management, 11(3), 195-208. doi:10.1037/1072-5245.11.3.195

Maxfield, L., \& Hyer, L. (2002). The relationship between efficacy and methodology in studies investigating EMDR treatment of PTSD. Journal of Clinical Psychology, 58(1), 23-41. doi:10.1002/jclp.1127

Moher, D., Shamseer, L., Clarke, M., Ghersi, D., Liberati, A., Petticrew, M., \& Stewart, L. A. (2015). Preferred reporting items for systematic review and meta-analysis protocols (PRISMA-P) 2015 statement. Systematic Reviews, 4(1), 1. doi:10.1186/2046-4053-4-1

Monson, C. M., Schnurr, P. P., Resick, P. A., Friedman, M. J., Young-Xu, Y., \& Stevens, S. P. (2006). Cognitive processing therapy for veterans with military-related posttraumatic stress disorder. Journal of Consulting and Clinical Psychology, 74(5), 898-907. doi:10.1037/0022006X.74.5.898

National Institute for Health Care Excellence. (2018). PostTraumatic stress disorder. Retrieved from https: / www. nice.org.uk/guidance/ng116/resources/posttraumaticstress-disorder-pdf-66141601777861

Nijdam, M. J., Gersons, B. P., Reitsma, J. B., De Jongh, A., \& Olff, M. (2012). Brief eclectic psychotherapy v. eye movement desensitisation and reprocessing therapy for post-traumatic stress disorder: Randomised controlled trial. The British Journal of Psychiatry, 200(3), 224-231. doi:10.1192/bjp.bp.111.099234

Power, K., McGoldrick, T., \& Brown, K. (2002). A controlled comparison of EMDR versus Exposure plus Cognitive Restructuring versus Wait List in the treatment of post traumatic stress disorder. Clinical Psychology and Psychotherapy, 9, 229-318. doi:10.1002/cpp.341

Puhan, M. A., Schünemann, H. J., Murad, M. H., Li, T., Brignardello-Petersen, R., Singh, J. A., . . Guyatt, G. H. (2014). A GRADE working group approach for rating the quality of treatment effect estimates from network meta-analysis. BMJ, 349, 5630. doi:10.1136/bmj.g5630

Rothbaum, B. O. (1997). A controlled study of eye movement desensitization and reprocessing in the treatment of posttraumatic stress disordered sexual assault victims. Bulletin of the Menninger Clinic, 61(3), 317.

Scheck, M. M., Schaeffer, J. A., \& Gillette, C. (1998). Brief psychological intervention with trawumatized young women: The efficacy of eye movement desensitization and reprocessing. Journal of Traumatic Stress, 11(1), 25-44. doi:http: / / dx.doi.org/10.1023 / a:1024400931106 
Schnurr, P. P., Friedman, M. J., Foy, D. W., Shea, M. T., Hsieh, F. Y., Lavori, P. W., . . Bernardy, N. C. (2003). Randomized trial of trauma-focused group therapy for posttraumatic stress disorder: Results from a department of veterans affairs cooperative study. Archives of General Psychiatry, 60(5), 481-489. doi:10.1001/archpsyc.60.5.481

Schulz, K. F., Altman, D. G., \& Moher, D. (2010). CONSORT 2010 statement: Updated guidelines for reporting parallel group randomised trials. BMC Medicine, 8(1), 18. doi:10.1186/1741-7015-8-18

Shapiro, E., \& Laub, B. (2015). Early EMDR intervention following a community critical incident: A randomized clinical trial. Journal of EMDR Practice and Research, 9(1), 17-27. doi:10.1891/1933-3196.9.1.17

Shapiro, E., Laub, B., \& Rosenblat, O. (2018). Early EMDR intervention following intense rocket attacks on a town: A randomised clinical trial. Clinical Neuropsychiatry: Journal of Treatment Evaluation, 15(3), 158-169.

Soberman, G. B., Greenwald, R., Rule, D. L., . (2002). A controlled study of eye movement desensitization and reprocessing (EMDR) for boys with conduct problems. Journal of Aggression, Maltreatment \& Trauma, 6(1), 217-236. doi:http: / dx.doi.org/10.1300/j146v06n01_11

Tarquinio, C., Rotonda, C., Houllé, W. A., Montel, S., Rydberg, J. A., Minary, L., \& Alla, F. (2016). Early psychological preventive intervention for workplace violence: A randomized controlled explorative and comparative study between EMDR-recent event and critical incident stress debriefing. Issues in Mental Health Nursing, 37(11), 787-799. doi:10.1080/01612840.2016.1224282

Taylor, S., Thordarson, D. S., Maxfield, L., Fedoroff, I. C., Lovell, K., \& Ogrodniczuk, J. (2013). Comparative efficacy, speed, and adverse effects of three PTSD treatments: Exposure therapy, EMDR, and relaxation training. Journal of Consulting and Clinical Psychology, 71(2), 330-338. doi:http:// dx.doi.org/10.1037/0022006x.71.2.330

Ter Heide, F. J. J., Mooren, T. T. M., Knipscheer, J. M., \& Kleber, R. J. (2014). EMDR with traumatized refugees: From experience-based to evidence-based practice. Journal of EMDR Practice and Research, 8(3), 147-156. doi: http: / / dx.doi.org/10.1891/1933-3196.8.3.147

Ter Heide, F. J., Mooren, T. M., Van de Schoot, R., De Jongh, A., \& Kleber, R. J. (2016). Eye movement desensitisation and reprocessing therapy v. stabilisation as usual for refugees: Randomised controlled trial. British Journal of Psychiatry, 311-318. doi:10.1192/bjp.bp.115.167775
Turner, R. M., Bird, S. M., \& Higgins, J. P. (2013). The impact of study size on meta-analyses: Examination of underpowered studies in Cochrane reviews. PLOS ONE, 8(3), e59202. doi:10.1371/journal.pone.0059202

Van den Berg, D. P., De Bont, P. A., Van der Vleugel, B. M., De Roos, C., De Jongh, A., \& Van der Gaag, M. (2015). Prolonged exposure vs eye movement desensitization and reprocessing vs waiting list for posttraumatic stress disorder in patients with a psychotic disorder: A randomized clinical trial. JAMA Psychiatry, 72(3), 259-267. doi:10.1001/jamapsychiatry.2014.2637

Van der Kolk, B. A., Spinazzola, J., Blaustein, M. E., Hopper, J. W., Hopper, E. K., Korn, D. L., ... Simpson, W. B. (2007). A randomized clinical trial of eye movement desensitization and reprocessing (EMDR), fluoxetine, and pill placebo in the treatment of posttraumatic stress disorder: Treatment effects and long-term maintenance. Journal of Clinical Psychiatry, 68(1), 37. doi:10.4088/JCP.v68n0105

Vaughan, K., Armstrong, M. S., Gold, R., O’Connor, N., Jenneke, W., \& Tarrier, N. (1994). A trial of eye movement desensitization compared to image habituation training and applied muscle relaxation in post-traumatic stress disorder. Journal of Behavior Therapy and Experimental Psychiatry, 25(4), 283-291.

Watson, C. G. (1990). Psychometric posttraumatic stress disorder measurement techniques: A review. Psychological Assessment: A Journal of Consulting and Clinical Psychology, 2(4), 460. doi:10.1037/1040-3590.2.4.460

Widiger, T. A., \& Samuel, D. B. (2005). Diagnostic categories or dimensions? A question for the diagnostic and statistical manual of mental disorders. Journal of Abnormal Psychology, 114(4), 494. doi:10.1037/0021-843X.114.4.494

Yurtsever, A., Konuk, E., Akyüz, T., Zat, Z., Tükel, F., Çetinkaya, M., \& Shapiro, E. (2018). An eye movement desensitization and reprocessing (EMDR) group intervention for syrian refugees with posttraumatic stress symptoms: Results of a randomized controlled trial. Frontiers in Psychology, 9, 493. doi:10.3389/fpsyg.2018.00493

Disclosure. Both authors receive financial remuneration for providing training in trauma therapies.

Correspondence regarding this article should be directed to Sarah Dominguez, School of Psychology and Exercise Science, Murdoch University, South Street, Murdoch, WA, Australia 6150. E-mail: s.dominguez@murdoch.edu.au 